International Mathematical Forum, 2, 2007, no. 15, 735 - 746

\title{
Submanifolds of an Almost Paraquaternionic Kähler Product Manifold
}

\author{
Gabriel Eduard Vîlcu \\ Department of Mathematics and Computer Science, \\ "Petroleum-Gas" University of Ploieşti, \\ Bulevardul Bucureşti, Nr. 39, Ploieşti, România \\ gvilcu@mail.upg-ploiesti.ro
}

\begin{abstract}
In this paper we define an almost paraquaternionic Kähler product manifold and study the geometry of its $F$-invariant submanifolds.

Mathematics Subject Classification: 53C15

Keywords: Paraquaternionic Kähler manifolds, paraquaternionic space forms

\section{Introduction}

The theory of paraquaternionic manifolds has been developing in recent years $([1],[5],[8],[9],[13])$, although its roots date back to 50's, in the work of P. Libermann ([11]).

The concept of quaternionic structure of the second kind has been defined as a triplet of endomorphisms of the tangent bundle $\left\{J_{1}, J_{2}, J_{3}\right\}$, in which $J_{1}$ is almost complex and $J_{2}$ and $J_{3}$ are almost product structures satisfying some relations of anti-commutation. S. Ianuş ([6]) showed the existence of such a structure on the tangent bundle of a manifold (without the condition that the dimension of the underlying manifold be a multiple of 4). Under certain conditions on the holonomy group of a metric adapted to such a structure, one arrives to the concept of paraquaternionic Kähler manifold. An important example in this theory is the paraquaternionic projective space as described by Blažić ([3]).

In the present note we show that the properties of $F$-invariant and $F$ antiinvariant submanifolds of a Kählerian product manifold ([2]) can be extended in the paraquaternionic geometry. In Section 2 we present the definition
\end{abstract}


and basic properties of paraquaternionic manifolds. In Section 3 we define the notion of almost paraquaternionic Kähler product manifold and give an example. In Section 4 we research the fundamental properties of $F$-invariant and $F$-antiinvariant submanifolds of an almost paraquaternionic Kähler product manifold.

\section{Paraquaternionic manifolds}

Let $M$ be a differentiable manifold of dimension $n$ and assume that there is a rank 3-subbundle $\sigma$ of $\operatorname{End}(T M)$ such that a local basis $\left\{J_{1}, J_{2}, J_{3}\right\}$ of sections of $\sigma$ exists satisfying:

$$
\left\{\begin{array}{c}
J_{\alpha}^{2}=-\epsilon_{\alpha} I d, \forall \alpha=\overline{1,3} \\
J_{1} J_{2}=-J_{2} J_{1}=-J_{3}
\end{array}\right.
$$

where $\epsilon_{1}=1, \epsilon_{2}=\epsilon_{3}=-1$.

Then the bundle $\sigma$ is called a paraquaternionic structure on $M$ and $\left\{J_{1}, J_{2}, J_{3}\right\}$ is called canonical local basis of $\sigma$.

A pseudo-Riemannian metric $g$ is said to be adapted to the paraquaternionic structure $\sigma$ if it satisfies:

$$
g\left(J_{\alpha} X, J_{\alpha} Y\right)=\epsilon_{\alpha} g(X, Y), \forall \alpha=\overline{1,3}
$$

for all vector fields $X, Y$ on $M$ and any local basis $\left\{J_{1}, J_{2}, J_{3}\right\}$ of $\sigma$. Moreover, $(M, \sigma, g)$ is said to be a paraquaternionic manifold.

It is clear that any paraquaternionic manifold is of dimension $n=4 \mathrm{~m}$ and any adapted metric is necessarily of neutral signature $(2 m, 2 m)$.

If the bundle $\sigma$ is parallel with respect to the Levi-Civita connection $\nabla$ of $g$, then $(M, \sigma, g)$ is said to be a paraquaternionic Kähler manifold. Equivalently, locally defined 1 -forms $\omega_{1}, \omega_{2}, \omega_{3}$ exist such that:

$$
\left\{\begin{array}{c}
\nabla_{X} J_{1}=-\omega_{3}(X) J_{2}+\omega_{2}(X) J_{3} \\
\nabla_{X} J_{2}=-\omega_{3}(X) J_{1}+\omega_{1}(X) J_{3} \\
\nabla_{X} J_{3}=\omega_{2}(X) J_{1}-\omega_{1}(X) J_{2}
\end{array}\right.
$$

for any vector field $X$ on $M$.

Let $(M, \sigma, g)$ be a paraquaternionic manifold. If $X \in T_{p} M, p \in M$, then the 4-plane $P Q(X)$ spanned by $\left\{X, J_{1} X, J_{2} X, J_{3} X\right\}$ is called a paraquaternionic 4-plane. A 2-plane in $T_{p} M$ spanned by $\{X, Y\}$ is called half-paraquaternionic if $P Q(X)=P Q(Y)$.

The sectional curvature for a half-paraquaternionic 2-plane is called paraquaternionic sectional curvature. A paraquaternionic Kähler manifold of constant paraquaternionic sectional curvature is said to be a paraquaternionic space form. 
We have the following important fundamental properties concerning paraquaternionic manifolds $([3],[5],[9],[13])$ :

1. Any paraquaternionic Kähler manifold is an Einstein manifold, provided that $\operatorname{dim} M>4$.

2. Any paraquaternionic space form is a locally symmetric space.

3. The curvature tensor of a paraquaternionic space form is:

$$
\begin{aligned}
R(X, Y) Z= & \frac{c}{4}\left\{g(Z, Y) X-g(X, Z) Y+\sum_{\alpha=1}^{3} \epsilon_{\alpha}\left[g\left(Z, J_{\alpha} Y\right) J_{\alpha} X\right.\right. \\
& \left.\left.-g\left(Z, J_{\alpha} X\right) J_{\alpha} Y+2 g\left(X, J_{\alpha} Y\right) J_{\alpha} Z\right]\right\}
\end{aligned}
$$

for all vector fields $X, Y, Z$ on $M$ and any local basis $\left\{J_{1}, J_{2}, J_{3}\right\}$ of $\sigma$.

Some recent results concerning the hypersurfaces of paraquaternionic manifolds we find in [7] .

\section{Almost Paraquaternionic Kähler product man- ifolds}

Let $M$ be a $n$-dimensional manifold endowed with a tensor $F$ of type $(1,1)$ such that:

$$
F^{2}=I d
$$

Then we say that $(M, F)$ is an almost product manifold and $F$ is called an almost product structure on $M$. Moreover, if $(M, F)$ admits a semi-Riemannian metric $g$ such that:

$$
g(F X, F Y)=g(X, Y),
$$

$\forall X, Y \in \Gamma(T M)$, then we say that $(M, F, g)$ is an almost product semiRiemannian manifold.

Definition 3.1 Let $(M, F, g)$ be an almost product semi-Riemannian manifold and assume that there is a rank 3-subbundle $\sigma$ of $\operatorname{End}(T M)$ such that a local basis $\left\{J_{1}, J_{2}, J_{3}\right\}$ exists of sections of $\sigma$ satisfying (1) and (2). Then $(M, F, \sigma, g)$ is said to be a paraquaternionic product manifold.

Definition 3.2 Let $(M, F, \sigma, g)$ be a product paraquaternionic manifold. If the Levi-Civita connection $\nabla$ of $g$ satisfies the following conditions:

$\left\{\begin{aligned}\left(\nabla_{X} J_{1}\right) Y & =k\left[-\omega_{3}(X) J_{2} Y+\omega_{2}(X) J_{3} Y-\omega_{3}(F X) J_{2}(F Y)+\omega_{2}(F X) J_{3}(F Y)\right] \\ \left(\nabla_{X} J_{2}\right) Y & =k\left[-\omega_{3}(X) J_{1} Y+\omega_{1}(X) J_{3} Y-\omega_{3}(F X) J_{1}(F Y)+\omega_{1}(F X) J_{3}(F Y)\right] \\ \left(\nabla_{X} J_{3}\right) Y & =k\left[\omega_{2}(X) J_{1} Y-\omega_{1}(X) J_{2} Y+\omega_{2}(F X) J_{1}(F Y)-\omega_{1}(F X) J_{2}(F Y)\right]\end{aligned}\right.$ 
for some non-zero constant $k$ and any vector field $X$ on $M, \omega_{1}, \omega_{2}, \omega_{3}$ being local 1-forms over the open for which $\left\{J_{1}, J_{2}, J_{3}\right\}$ is a local basis of $\sigma$, then $(M, F, \sigma, g)$ is said to be an almost paraquaternionic Kähler product manifold.

\section{Example 3.3}

Let $\left(M_{1}, g_{1}, \sigma_{1}\right)$ be a paraquaternionic Kähler manifold. Then, in any coordinate neighborhood $U_{1}$ of $M_{1}$, there exists a local bases $\left\{J_{1}^{(1)}, J_{2}^{(1)}, J_{3}^{(1)}\right\}$ of $\sigma_{1}$ satisfying (1) and (2). Moreover, the Levi-Civita connection $\nabla^{(1)}$ of $g_{1}$ satisfies the following conditions:

$$
\left\{\begin{array}{c}
\nabla_{X}^{(1)} J_{1}^{(1)}=-\omega_{3}^{(1)}(X) J_{2}^{(1)}+\omega_{2}^{(1)}(X) J_{3}^{(1)} \\
\nabla_{X}^{(1)} J_{2}^{(1)}=-\omega_{3}^{(1)}(X) J_{1}^{(1)}+\omega_{1}^{(1)}(X) J_{3}^{(1)} \\
\nabla_{X}^{(1)} J_{3}^{(1)}=\omega_{2}^{(1)}(X) J_{1}^{(1)}-\omega_{1}^{(1)}(X) J_{2}^{(1)}
\end{array}\right.
$$

for any vector field $X$ on $M_{1}, \omega_{1}^{(1)}, \omega_{2}^{(1)}, \omega_{3}^{(1)}$ being local 1-forms over the open for which $\left\{J_{1}^{(1)}, J_{2}^{(1)}, J_{3}^{(1)}\right\}$ is a local basis of $\sigma_{1}$.

Let $\left(M_{2}, g_{2}, \sigma_{2}\right)$ be an another paraquaternionic Kähler manifold. Then, in any coordinate neighborhood $U_{2}$ of $M_{2}$, there exists a local basis $\left\{J_{1}^{(2)}, J_{2}^{(2)}, J_{3}^{(2)}\right\}$ of $\sigma_{2}$ satisfying (1) and (2). Moreover, the Levi-Civita connection $\nabla^{(2)}$ of $g_{2}$ satisfies the following conditions:

$$
\left\{\begin{array}{c}
\nabla_{X}^{(2)} J_{1}^{(2)}=-\omega_{3}^{(2)}(X) J_{2}^{(2)}+\omega_{2}^{(2)}(X) J_{3}^{(2)} \\
\nabla_{X}^{(2)} J_{2}^{(2)}=-\omega_{3}^{(2)}(X) J_{1}^{(2)}+\omega_{1}^{(2)}(X) J_{3}^{(2)} \\
\nabla_{X}^{(2)} J_{3}^{(2)}=\omega_{2}^{(2)}(X) J_{1}^{(2)}-\omega_{1}^{(2)}(X) J_{2}^{(2)}
\end{array}\right.
$$

for any vector field $X$ on $M_{2}, \omega_{1}^{(2)}, \omega_{2}^{(2)}, \omega_{3}^{(2)}$ being local 1-forms over the open for which $\left\{J_{1}^{(2)}, J_{2}^{(2)}, J_{3}^{(2)}\right\}$ is a local basis of $\sigma_{2}$.

Let $M=M_{1} \times M_{2}$ be the product manifold of $M_{1}$ and $M_{2}$. We define a semi-Riemannian metric $g$ on $M$ by:

$$
g(X, Y)=g_{1}\left(\Pi_{1} X, \Pi_{1} Y\right)+g_{2}\left(\Pi_{2} X, \Pi_{2} Y\right)
$$

for any vector fields $X$ and $Y$ on $M$, where $\Pi_{1}$ and $\Pi_{2}$ denote the projection mappings of $\Gamma\left(T\left(M_{1} \times M_{2}\right)\right)$ to $\Gamma\left(T\left(M_{1}\right)\right)$ and $\Gamma\left(T\left(M_{2}\right)\right)$, respectively, that is:

$$
\Pi_{1}: \Gamma(T M) \rightarrow \Gamma\left(T M_{1}\right), \Pi_{2}: \Gamma(T M) \rightarrow \Gamma\left(T M_{2}\right) .
$$

We define now:

$$
F=\Pi_{1}-\Pi_{2}
$$

and we can easily see that $(M, F, g)$ is an almost product semi-Riemannian manifold. 
We define the endomorphisms of tangent space of $M$ by:

$$
J_{\alpha} X=J_{\alpha}^{(1)} \Pi_{1} X+J_{\alpha}^{(2)} \Pi_{2} X, \forall \alpha=\overline{1,3}
$$

for any vector field $X$ on $M$, where $\left\{J_{\alpha}^{(1)}\right\}_{\alpha=\overline{1,3}}$ and $\left\{J_{\alpha}^{(2)}\right\}_{\alpha=\overline{1,3}}$ are local basis of $\sigma_{1}$ and $\sigma_{2}$, respectively.

If we consider the vector bundle $\sigma$ over $M$ generated by $\left\{J_{\alpha}\right\}_{\alpha=\overline{1,3}}$, then we have that $(M, g, \sigma)$ is a paraquaternionic manifold(see [12]).

Now, for any local coordinate neighborhood $U_{1} \times U_{2}$ of $M$ we define local 1 -form $\omega_{1}, \omega_{2}, \omega_{3}$ by:

$$
\omega_{\alpha}(X)=\omega_{\alpha}^{(1)}\left(\Pi_{1} X\right)+\omega_{\alpha}^{(2)}\left(\Pi_{2} X\right), \forall \alpha=\overline{1,3},
$$

for any vector field $X$ on $M$.

We remark that the Levi-Civita connection $\nabla$ on $M$ is:

$$
\nabla_{X} Y=\nabla_{\Pi_{1} X}^{(1)} \Pi_{1} Y+\nabla_{\Pi_{2} X}^{(2)} \Pi_{2} Y
$$

for any vector fields $X$ and $Y$ on $M$.

We have now:

$$
\begin{aligned}
\left(\nabla_{X} J_{1}\right) Y= & \nabla_{X} J_{1} Y-J_{1}\left(\nabla_{X} Y\right) \\
& =\nabla_{\Pi_{1} X}^{(1)} \Pi_{1} J_{1} Y+\nabla_{\Pi_{2} X}^{(2)} \Pi_{2} J_{1} Y-J_{1} \nabla_{\Pi_{1} X}^{(1)} \Pi_{1} Y-J_{1} \nabla_{\Pi_{2} X}^{(2)} \Pi_{2} Y \\
& =\nabla_{\Pi_{1} X}^{(1)} J_{1}^{(1)} \Pi_{1} Y+\nabla_{\Pi_{2} X}^{(2)} J_{1}^{(2)} \Pi_{2} Y-J_{1}^{(1)} \nabla_{\Pi_{1} X}^{(1)} \Pi_{1} Y-J_{1}^{(2)} \nabla_{\Pi_{2} X}^{(2)} \Pi_{2} \\
& =\left(\nabla_{\Pi_{1} X}^{(1)} J_{1}^{(1)}\right)\left(\Pi_{1} Y\right)+\left(\nabla_{\Pi_{2} X}^{(2)} J_{1}^{(2)}\right)\left(\Pi_{2} Y\right) .
\end{aligned}
$$

Thus, taking account of (8) and (9), we obtain:

$$
\begin{aligned}
\left(\nabla_{X} J_{1}\right) Y= & -\omega_{3}^{(1)}\left(\Pi_{1} X\right) J_{2}^{(1)}\left(\Pi_{1} Y\right)+\omega_{2}^{(1)}\left(\Pi_{1} X\right) J_{3}^{(1)}\left(\Pi_{1} Y\right) \\
& -\omega_{3}^{(2)}\left(\Pi_{2} X\right) J_{2}^{(2)}\left(\Pi_{2} Y\right)+\omega_{2}^{(2)}\left(\Pi_{2} X\right) J_{3}^{(2)}\left(\Pi_{2} Y\right) .
\end{aligned}
$$

On the other hand, by using (13) we obtain:

$$
\begin{aligned}
-\omega_{3}(X) J_{2} Y+ & \omega_{2}(X) J_{3} Y-\omega_{3}(F X) J_{2}(F Y)+\omega_{2}(F X) J_{3}(F Y) \\
& =2\left\{-\omega_{3}^{(1)}\left(\Pi_{1} X\right) J_{2}^{(1)}\left(\Pi_{1} Y\right)+\omega_{2}^{(1)}\left(\Pi_{1} X\right) J_{3}^{(1)}\left(\Pi_{1} Y\right)\right. \\
& \left.-\omega_{3}^{(2)}\left(\Pi_{2} X\right) J_{2}^{(2)}\left(\Pi_{2} Y\right)+\omega_{2}^{(2)}\left(\Pi_{2} X\right) J_{3}^{(2)}\left(\Pi_{2} Y\right)\right\} .
\end{aligned}
$$

From (16) and (17) we deduce:

$$
\left(\nabla_{X} J_{1}\right) Y=\frac{1}{2}\left[-\omega_{3}(X) J_{2} Y+\omega_{2}(X) J_{3} Y-\omega_{3}(F X) J_{2}(F Y)+\omega_{2}(F X) J_{3}(F Y)\right]
$$

and similarly we obtain:

$$
\left(\nabla_{X} J_{2}\right) Y=\frac{1}{2}\left[-\omega_{3}(X) J_{1} Y+\omega_{1}(X) J_{3} Y-\omega_{3}(F X) J_{1}(F Y)+\omega_{1}(F X) J_{3}(F Y)\right]
$$


and

$\left(\nabla_{X} J_{3}\right) Y=\frac{1}{2}\left[\omega_{2}(X) J_{1} Y-\omega_{1}(X) J_{2} Y+\omega_{2}(F X) J_{1}(F Y)-\omega_{1}(F X) J_{2}(F Y)\right]$.

Thus, we conclude that $(M, F, \sigma, g)$ is an almost paraquaternionic Kähler product manifold.

We remark that if $M_{1}\left(c_{1}\right)$ and $M_{2}\left(c_{2}\right)$ are paraquaternionic space forms with constant paraquaternionic sectional curvatures $c_{1}$ and $c_{2}$, respectively, then the curvature tensor $R$ of paraquaternionic Kähler product manifold $M=M_{1} \times M_{2}$ is given by:

$$
\begin{aligned}
R(X, Y) Z= & \frac{c_{1}+c_{2}}{16}\{g(Z, Y) X-g(X, Z) Y+g(Z, F Y) F X-g(X, F Z) F Y \\
& +\sum_{\alpha=1}^{3} \epsilon_{\alpha}\left[g\left(Z, J_{\alpha} Y\right) J_{\alpha} X-g\left(Z, J_{\alpha} X\right) J_{\alpha} Y+2 g\left(X, J_{\alpha} Y\right) J_{\alpha} Z\right. \\
& \left.\left.+g\left(Z, F J_{\alpha} Y\right) F J_{\alpha} X-g\left(Z, F J_{\alpha} X\right) F J_{\alpha} Y+2 g\left(X, F J_{\alpha} Y\right) F J_{\alpha} Z\right]\right\} \\
& +\frac{c_{1}-c_{2}}{16}\{g(Z, F Y) X-g(X, F Z) Y+g(Z, Y) F X-g(X, Z) F Y \\
& +\sum_{\alpha=1}^{3} \epsilon_{\alpha}\left[g\left(Z, F J_{\alpha} Y\right) J_{\alpha} X-g\left(Z, F J_{\alpha} X\right) J_{\alpha} Y+2 g\left(X, F J_{\alpha} Y\right) J_{\alpha} Z\right. \\
& \left.\left.+g\left(Z, J_{\alpha} Y\right) F J_{\alpha} X-g\left(Z, J_{\alpha} X\right) F J_{\alpha} Y+2 g\left(X, J_{\alpha} Y\right) F J_{\alpha} Z\right]\right\}
\end{aligned}
$$

for all vector fields $X, Y, Z$ on $M$, where $F$ is the almost product structure on $M$ given by (11) and $J_{\alpha}$ is given by (12) (see [12]).

\section{F-invariant submanifolds of a paraquater- nionic product manifold}

Let $(\bar{M}, \bar{g})$ be a $n$-dimensional semi-Riemannian manifold and $(M, g)$ be a $m$ dimensional semi-Riemannian submanifold of $\bar{M}$. If the curvature tensor $R$ of $M$ satisfies the following condition:

$$
R(X, Y) Z \in \Gamma(T M), \forall X, Y, Z \in \Gamma(T M)
$$

then $M$ is said to be a curvature-invariant submanifold of $\bar{M}$.

Definition 4.1 Let $(\bar{M}, F, \sigma, \bar{g})$ be a n-dimensional paraquaternionic product manifold and $(M, g)$ be a m-dimensional semi-Riemannian submanifold of $\bar{M}$. i. If $F\left(T_{p} M\right) \subset T_{p} M, \forall p \in M$, then $M$ is said to be F-invariant submanifold of paraquaternionic product manifold $\bar{M}$.

ii. If $F\left(T_{p} M\right) \subset T_{p} M^{\perp}, \forall p \in M$, where $T_{p} M^{\perp}$ is the normal space of $T_{p} M$ in $T_{p} \bar{M}, p \in M$, then $M$ is said to be $F$-antiinvariant submanifold of paraquaternionic product manifold $\bar{M}$. 
Definition 4.2 Let $(\bar{M}, F, \sigma, \bar{g})$ be a paraquaternionic product manifold. A semi-Riemannian submanifold $(M, g)$ of $\bar{M}$ is called paraquaternionic submanifold if:

$$
J\left(T_{p} M\right) \subset T_{p} M, \forall J \in \sigma_{p}, \forall p \in M .
$$

Theorem 4.3 Let $\bar{M}=\bar{M}_{1} \times \bar{M}_{2}$ be the paraquaternionic product manifold of two paraquaternionic space forms $\bar{M}_{1}(c)$ and $\bar{M}_{2}(c), c \neq 0$. If $M$ is a curvature-invariant paraquaternionic submanifold of $\bar{M}$, then $M$ is $F$-invariant or F-antiinvariant.

Proof. From (18), because $c_{1}=c_{2}=c$, we obtain that the curvature tensor of $\bar{M}$ is given by:

$$
\begin{aligned}
\bar{R}(X, Y) Z= & \frac{c}{8}\{\bar{g}(Z, Y) X-\bar{g}(X, Z) Y+\bar{g}(Z, F Y) F X-\bar{g}(X, F Z) F Y \\
& +\sum_{\alpha=1}^{3} \epsilon_{\alpha}\left[\bar{g}\left(Z, J_{\alpha} Y\right) J_{\alpha} X-\bar{g}\left(Z, J_{\alpha} X\right) J_{\alpha} Y+2 \bar{g}\left(X, J_{\alpha} Y\right) J_{\alpha} Z \quad(20)\right. \\
& \left.\left.+\bar{g}\left(Z, F J_{\alpha} Y\right) F J_{\alpha} X-\bar{g}\left(Z, F J_{\alpha} X\right) F J_{\alpha} Y+2 \bar{g}\left(X, F J_{\alpha} Y\right) F J_{\alpha} Z\right]\right\} .
\end{aligned}
$$

On the other hand, $M$ is a paraquaternionic submanifold of $\bar{M}$ and thus we have:

$$
\begin{aligned}
& g(Z, F Y) F X-g(X, F Z) F Y+\sum_{\alpha=1}^{3} \epsilon_{\alpha}\left[g\left(Z, F J_{\alpha} Y\right) F J_{\alpha} X\right. \\
& \left.-g\left(Z, F J_{\alpha} X\right) F J_{\alpha} Y+2 g\left(X, F J_{\alpha} Y\right) F J_{\alpha} Z\right] \in T_{p} M
\end{aligned}
$$

$\forall X, Y, Z \in T_{p} M, \forall p \in M$.

If we take now $Z=X$ in (21) we obtain:

$$
\begin{aligned}
& g(X, F Y) F X-g(X, F X) F Y+3\left[g\left(F X, J_{1} Y\right) F J_{1} X\right. \\
& \left.-g\left(F X, J_{2} Y\right) F J_{2} X-g\left(F X, J_{3} Y\right) F J_{3} X\right] \in T_{p} M .
\end{aligned}
$$

Replacing $X$ by $J_{1} X, J_{2} X$ and $J_{3} X$ in (22) we get:

$$
\begin{aligned}
& \quad g\left(J_{1} X, F Y\right) F J_{1} X-g(X, F X) F Y+3[-g(F X, Y) F X \\
& \left.\quad-g\left(F X, J_{3} Y\right) F J_{3} X-g\left(F X, J_{2} Y\right) F J_{2} X\right] \in T_{p} M, \\
& g\left(J_{2} X, F Y\right) F J_{2} X+g(X, F X) F Y+3\left[g\left(F X, J_{3} Y\right) F J_{3} X\right. \\
& \left.+g(F X, Y) F X-g\left(F X, J_{1} Y\right) F J_{1} X\right] \in T_{p} M
\end{aligned}
$$

and

$$
\begin{aligned}
& g\left(J_{3} X, F Y\right) F J_{3} X+g(X, F X) F Y+3\left[g\left(F X, J_{2} Y\right) F J_{2} X\right. \\
& \left.-g\left(F X, J_{1} Y\right) F J_{1} X+g(F X, Y) F X\right] \in T_{p} M
\end{aligned}
$$


Adding (23), (24) and (25) we derive:

$$
\begin{array}{r}
g(X, F X) F Y+3 g(F X, Y) F X-\left[7 g\left(F X, J_{1} Y\right) F J_{1} X\right. \\
\left.+g\left(F X, J_{2} Y\right) F J_{2} X+g\left(F X, J_{3} Y\right) F J_{3} X\right] \in T_{p} M .
\end{array}
$$

From (23) and (26) we obtain:

$$
g(X, F X) F Y+3 g(F X, Y) F X-5 g\left(F X, J_{1} Y\right) F J_{1} X \in T_{p} M .
$$

On the other hand, from (22) and (23) we have:

$$
g(F X, Y) F X+g\left(F X, J_{1} Y\right) F J_{1} X \in T_{p} M .
$$

From (27) and (28) it follows that:

$$
g(X, F X) F Y+8 g(F X, Y) F X \in T_{p} M
$$

and putting now $Y=X$ in (29) we derive:

$$
g(X, F X) F X \in T_{p} M .
$$

The relation (30) implies that $F X \in T_{p} M$ or $g(X, F X)=0$. In the last situation, from (29) we obtain:

$$
g(F X, Y) F X \in T_{p} M
$$

and consequently we have $F X \in T_{p} M$ or $F X \in T_{p} M^{\perp}$ for any vector $X \in$ $T_{p} M$. Thus we have $F\left(T_{p} M\right) \subset T_{p} M$ or $F\left(T_{p} M\right) \subset T_{p} M^{\perp}$ for each point $p \in M$ and the proof is now complete.

Theorem 4.4 Let $\bar{M}=\bar{M}_{1} \times \bar{M}_{2}$ be the paraquaternionic product manifold of two paraquaternionic space forms $\bar{M}_{1}(c)$ and $\bar{M}_{2}(c), c \neq 0$. If $M$ is a totally geodesic submanifold of $\bar{M}$, then $M$ is $F$-invariant or $F$-antiinvariant.

Proof. Because $M$ is a totally geodesic submanifold of $\bar{M}$ it follows that $M$ is a curvature-invariant ant the proof is now complete from Theorem 4.3.

Let us assume that $\left(\bar{M}=\bar{M}_{1} \times \bar{M}_{2}, F, \sigma, \bar{g}\right)$ be a $n$-dimensional paraquaternionic product manifold and $(M, g)$ be an $m$-dimensional semi-Riemannian nondegenerate submanifolds of $\bar{M}$. For any $X \in \Gamma(T M)$ we have decomposition:

$$
F X=f X+n X
$$

where $f X$ is the tangential part of $F X$ and $n X$ is the normal part of $F X$. Similarly, for any vector $\xi \in \Gamma\left(T M^{\perp}\right)$ we have:

$$
F \xi=t \xi+s \xi
$$


where $t \xi$ is the tangential part of $F \xi$ and $s \xi$ is the normal part of $F \xi$. From (32) and (33) we obtain:

$$
F^{2} X=f^{2} X+n(f X)+t(n X)+s(n X) .
$$

From (5) and (34) we deduce:

$$
f^{2} X=X-s(n X) .
$$

On the other hand, for $\forall X, Y \in \Gamma(T M)$, from (31) we derive:

$$
\bar{g}(F X, F Y)=g(f X, f Y)+\bar{g}(n X, n Y) .
$$

From (6) and (36) it follows now that we have:

$$
g(f X, f Y)=g(X, Y)-\bar{g}(n X, n Y) .
$$

Theorem 4.5 Let $\left(\bar{M}=\bar{M}_{1} \times \bar{M}_{2}, F, \sigma, \bar{g}\right)$ be a paraquaternionic Kähler product manifold. If $M$ is a F-invariant nondegenerate submanifold of $\bar{M}$, then $M=M_{1} \times M_{2}$, where $M_{1}$ and $M_{2}$ are submanifolds of $\bar{M}_{1}$ and $\bar{M}_{2}$, respectively. Moreover, $M_{1}$ and $M_{2}$ are totally geodesic submanifolds of $M$.

Proof. Because $M$ is a $F$-invariant submanifold of $\bar{M}$, we have:

$$
n X=0, \forall X \in \Gamma(T M) .
$$

From (35), (36) and (38) we obtain:

$$
f^{2} X=X
$$

and

$$
g(f X, f Y)=g(X, Y)
$$

for any $X, Y \in \Gamma(T M)$.

Thus we conclude that $(f, g)$ is an almost semi-Riemannian product structure on $M$ and consequently the vertical distribution $T_{1}$ and the horizontal distribution $T_{2}$ are given by:

$$
T_{1}=\{X \in \Gamma(T M) \mid f X=X\}
$$

and

$$
T_{2}=\{X \in \Gamma(T M) \mid f X=-X\} .
$$

Let $M_{1}$ and $M_{2}$ be the integral manifolds of the vertical distribution $T_{1}$ and the horizontal distribution $T_{2}$, respectively.

Because we have:

$$
\nabla_{Y} f X=f \nabla_{Y} X
$$


we deduce:

$$
f \nabla_{Y} X_{1}=\nabla_{Y} f X_{1}=\nabla_{Y} X_{1}
$$

for all $X_{1} \in T_{1}$ and $Y \in \Gamma(T M)$.

Thus we conclude that the distribution $T_{1}$ is parallel.

On the other hand, for any $X_{1}, Y_{1} \in T_{1}$ we have:

$$
\begin{aligned}
f\left[X_{1}, Y_{1}\right]= & f \nabla_{X_{1}} Y_{1}-f \nabla_{Y_{1}} X_{1}=\nabla_{X_{1}} f Y_{1}-\nabla_{Y_{1}} f X_{1} \\
& =\nabla_{X_{1}} Y_{1}-\nabla_{Y_{1}} X_{1}=\left[X_{1}, Y_{1}\right]
\end{aligned}
$$

and thus we conclude that the distribution $T_{1}$ is involutive.

In the same way we obtain that the distribution $T_{2}$ is parallel and involutive.

Now, taking into account that $T_{1}$ and $T_{2}$ are orthogonal distributions and $\nabla$ is the Levi-Civita connection, we obtain:

$$
g\left(\nabla_{X_{1}} Y_{1}, Z_{2}\right)=-g\left(Y_{1}, \nabla_{X_{1}} Z_{2}\right)=0
$$

for any $X_{1}, Y_{1} \in T_{1}$ and $Z_{2} \in T_{2}$.

Hence $M_{1}$ is a totally geodesic submanifold of $M$ and similarly we have $M_{2}$ is a totally geodesic submanifold of $M$.

On the other hand, for any $X_{1} \in T_{1}$ we have:

$$
\begin{gathered}
\Pi_{1} X_{1}=\frac{1}{2}\left(\Pi_{1}+\Pi_{2}+\Pi_{1}-\Pi_{2}\right)\left(X_{1}\right) \\
=\frac{1}{2}(I d+F)\left(X_{1}\right)=X_{1}
\end{gathered}
$$

and

$$
\begin{aligned}
\Pi_{2} X_{1}= & \frac{1}{2}\left[\Pi_{1}+\Pi_{2}-\left(\Pi_{1}-\Pi_{2}\right)\right]\left(X_{1}\right) \\
& =\frac{1}{2}(I d-F)\left(X_{1}\right)=0 .
\end{aligned}
$$

From (47) and (48) we conclude that $M_{1}$ is a submanifold of $\bar{M}_{1}$. In the same way, $M_{2}$ is a submanifold of $\bar{M}_{2}$.

The proof is now complete.

Theorem 4.6 Let $\left(\bar{M}=\bar{M}_{1} \times \bar{M}_{2}, F, \sigma, \bar{g}\right)$ be a paraquaternionic Kähler product manifold. If $M$ is a $F$-invariant paraquaternionic nondegenerate submanifold of $\bar{M}$, then $M=M_{1} \times M_{2}$, where $M_{1}$ and $M_{2}$ are paraquaternionic submanifolds of $\bar{M}_{1}$ and $\bar{M}_{2}$, respectively.

Proof. From the above Theorem we have that $M=M_{1} \times M_{2}$, where $M_{1}$ and $M_{2}$ are submanifolds of $\bar{M}_{1}$ and $\bar{M}_{2}$, respectively. 
Let $J_{1}, J_{2}, J_{3}$ be a local basis of $\sigma$ given by (12). If $X \in T_{p} M_{1}, p \in M_{1}$, then we have:

$$
J_{\alpha} X=J_{\alpha}^{(1)} \Pi_{1} X+J_{\alpha}^{(2)} \Pi_{2} X=J_{\alpha}^{(1)} \Pi_{1} X \in T_{p} \bar{M}_{1} \cap T_{p} M=T_{p} M
$$

$\forall \alpha=\overline{1,3}$. Consequently we deduce that $M_{1}$ ia a paraquaternionic submanifolds of $\bar{M}_{1}$ and similarly $M_{2}$ is a paraquaternionic submanifolds of $\bar{M}_{2}$.

\section{ACKNOWLEDGEMENTS.}

This work was supported by the Romanian Ministry of Education and Research under Grant CEEX 2250/2006.

\section{References}

[1] D. Alekseevsky, Y. Kamishima, Quaternionic and para-quaternionic CR structure on $(4 \mathrm{n}+3)$-dimensional manifolds, Central European J. Math, 2(5) (2004), 732-753.

[2] M. Atceken, F-invariant submanifolds of Kaehlerian product manifold, Turk. J. Math, 28 (2004), 367-381.

[3] N. Blažić, Para-quaternionic projective spaces and pseudo Riemannian geometry, Publ. Inst. Math, 60 (1996), 101-107.

[4] B.Y. Chen, Geometry of submanifolds and its applications, Sci. University, Tokio, 1981.

[5] E. Garcia Rio, Y.Matsushita, R. Vasquez-Lorenzo, Paraquaternionic Kähler manifolds, Rocky Mountain Journal of Math, 31 (2001), 237-260.

[6] S. Ianus, Sulle strutture canoniche dello spazio fibrato tangente di una varieta riemanniana, Rend. Mat., 6 (1973), 75-96.

[7] S. Ianuş, R. Mazzocco, G.E. Vîlcu, Real lightlike hypersurfaces of paraquaternionic Kähler manifolds", Mediterr. J. Math., 3 (2006), 581592.

[8] S. Ivanov, I. Minchev, S. Zamkovoy, Twistor and Reflector Spaces of Almost Para-Quaternionic Manifolds, e-print, math. DG/0511657.

[9] S. Ivanov, S. Zamkovoy, Para-hermitian and para-quaternionic manifolds, Differential Geom. Appl., 23 (2005), 205-234.

[10] T.H. Kang, H.C. Nam, Submanifolds of an almost quaternionic Kaehler product manifold, Bull. Korean Math. Soc., 34 (1997), 635-665. 
[11] P. Libermann, Sur les structures presque quaternioniennes de deuxième espéce, C.R. Acad. Sc. Paris, 234 (1952), 1030-1032.

[12] G.E. Vîlcu, Product of paraquaternionic Kähler manifolds, Bull. Math. Soc. Sc. Math. Roumanie, 48 (2005), 345-355.

[13] S. Vukmirović, Para-quaternionic reduction, e-print, math. DG/03044424.

[14] K. Yano, M. Kon, Submanifolds of Kaehlerian product manifolds, Memorie Accademia Nazionale dei Lincei, Vol. CCCLXXVI (1979), 267-292.

Received: July 6, 2006 